\title{
Synchronous small and large bowel cancer developing after pelvic irradiation
}

\author{
H GAJRAJ, D R DAVIES, AND B T JACKSON \\ From St Thomas's Hospital, London
}

Summary This case report supports the belief that there is a causal relationship between therapeutic radiation and subsequent bowel malignancy.

\section{Case report}

A woman, aged 42 years, with a stage III squamous cell carcinoma of the cervix was treated with internal and external cobalt 60 irradiation for 45 days. The combined dose to the cervix (point A) was 72 Gy $(7200 \mathrm{rad}$ ) and to areas $5 \mathrm{~cm}$ from the cervix (point B) $52.8 \mathrm{~Gy}(5280 \mathrm{rad})$. She remained well for six years, when she developed painless haematuria and clot retention. After an intravenous urogram and cystoscopy, radiation cystitis was diagnosed and the bleeding was attributed to areas of telangiectasia. The bleeding stopped spontaneously. Fourteen years after treatment, she remained free of recurrent disease and was discharged from outpatient care.

Twenty years after radiation therapy, aged 62 years, she was admitted to hospital with intestinal obstruction. At operation the sigmoid colon, upper rectum, caecum and terminal ileum were thickened and adherent to surrounding structures and this was thought to be caused by radiation exposure.

The terminal ileum was strictured. There was a pinhole perforation on the anterior wall of the grossly distended caecum. The large bowel was obstructed at the rectosigmoid junction where a small mobile mass was noted. In view of the patient's poor general condition, a tube caecostomy was fashioned through the caecal perforation and a loop ileostomy raised proximal to the ileal stricture.

Address for correspondence: Mr B T Jackson, St Thomas's Hospital, I ambeth Palace Road. I.ondon SI:1 7I:H.

Received for publication 2 July 1987.
Two days' later, when the patient's general condition had improved, the diseased small and large bowel were excised, preserving the normal transverse colon. The right colon and $35 \mathrm{~cm}$ of terminal ileum were removed, an end ileostomy fashioned and the transverse colon brought out as a mucous fistula. The sigmoid colon and upper rectum were also excised, oversewing the rectal stump and bringing out the descending colon as a second mucous fistula. It was hoped that restoration of continuity might be possible at a later stage. She made a satisfactory recovery and was discharged four weeks' later.

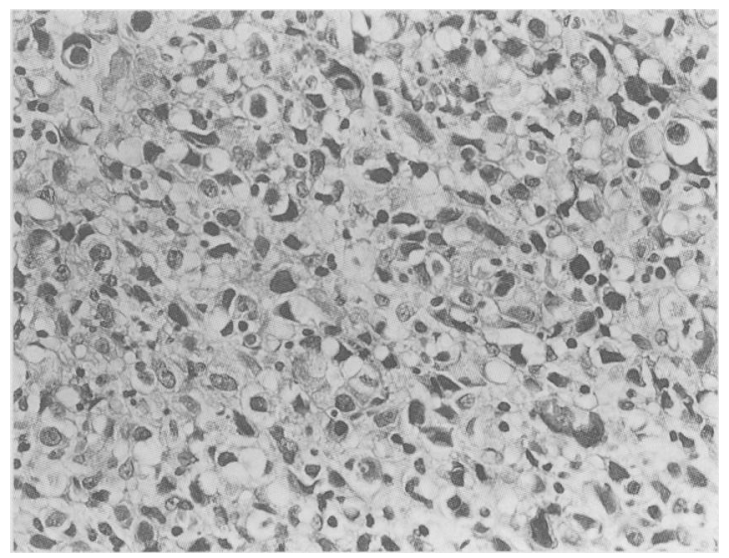

Fig. 1 Tumour in large intestine. This is an area of poorly differentiated adenocarcinoma with some signet ring forms. ( $\mathrm{H} \& \mathrm{E})$ 


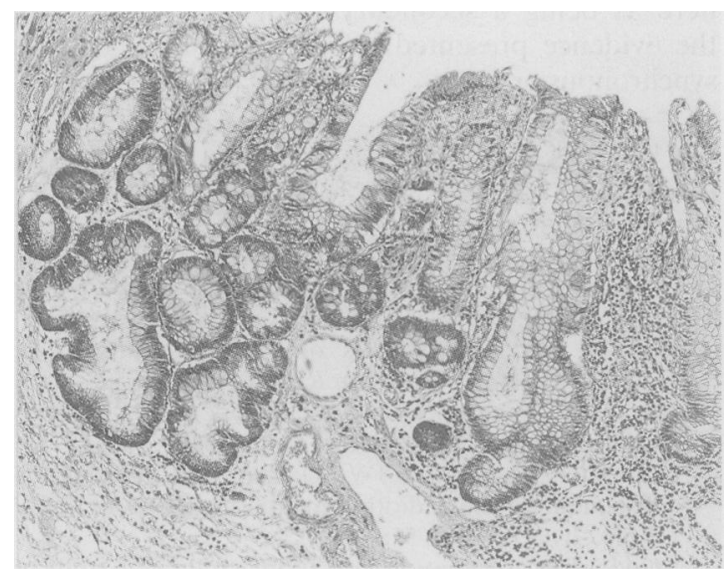

Fig. 2 Large bowel mucosa adjacent to carcinoma showing distorted glands with nuclear hyperchromasia and atypia. This is most marked in the glands on the left of the field. ( $H \& E)$

Histological examination showed a Dukes' stage C adenocarcinoma of the rectum. The tumour was poorly differentiated with a signet ring cell type in some areas (Fig. 1) and moderately differentiated with gland formation in others. In the adjoining bowel, the mucosa showed distorted glands with nuclear atypia (Fig. 2). Telangiectasia were present and larger arterioles showed endarteritis obliterans. Vacuolated 'radiation' fibroblasts, with occasional multinucleate forms, were present in the connective tissue.

The annular stricture of the terminal ileum was an ulcerating, moderately differentiated adenocar-

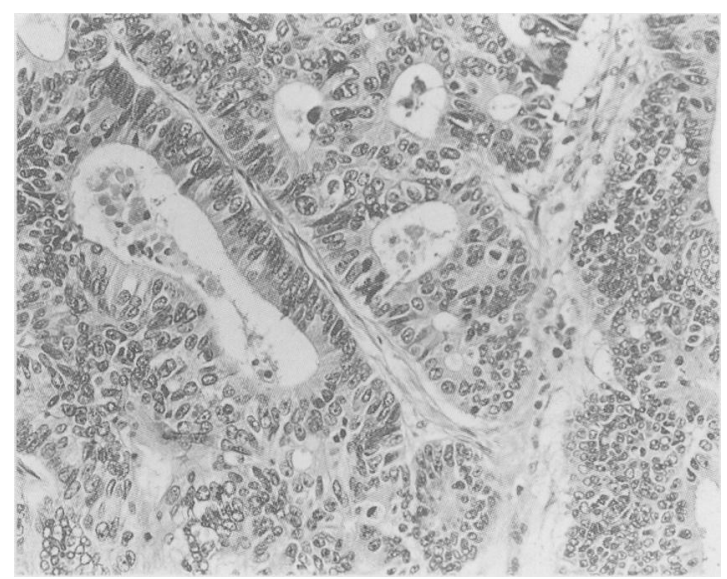

Fig. 3 Tumour in small intestine. This is an area of moderately differentiated adenocarcinoma with gland formation. (H\& E)

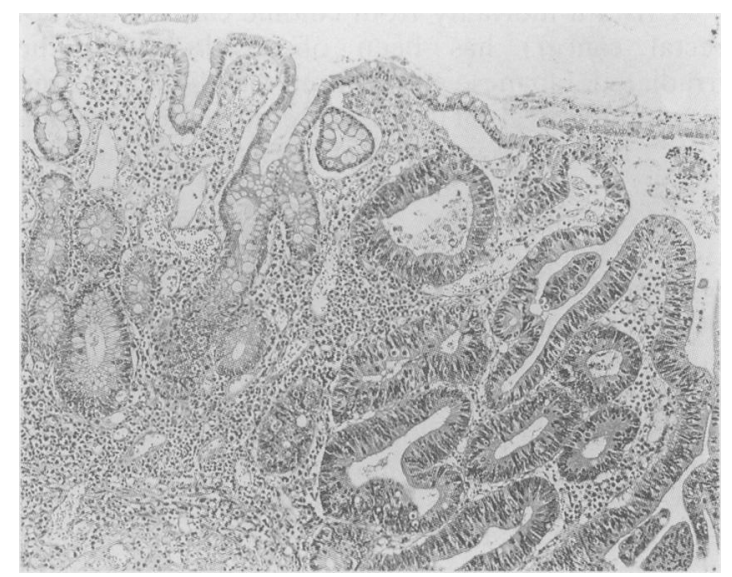

Fig. 4 Small intestinal mucosa. There is loss of the normal villous pattern (on the left of the picture). There are neoplastic glands in the mucosa (right of picture). (H\& E)

cinoma (Fig. 3) penetrating right through the bowel wall and with metastatic tumour present in a lymph node of the mesentery. Intramucosal carcinoma was present in adjacent mucosa which also showed loss of the normal villous pattern in the immediate area of the tumour (Fig. 4). Telangiectasia, arterial intimal thickening (less marked than in the colon) and vacuolated fibroblasts with occasional multinucleate forms were present.

Five months' later, laparotomy was performed with the intention of closing the ileostomy but multiple metastatic deposits were present in the mesentery and the liver.

\section{Discussion}

Evidence that supports therapeutic radiation as a cause of colonic cancer comes from cohort studies and from case reports.

Cohorts of women who have received pelvic irradiation for cervical cancer provide conflicting evidence for an increased risk of large bowel malignancy. " Such studies are complicated by the fact that women with gynaecological malignancy are believed to be at an increased risk of developing a second primary malignancy of the large bowel.' Groups of women receiving pelvic irradiation for benign gynaecological disorders also give conflicting results. ${ }^{+ \text {th }}$ Short follow up periods and low response rates with potential for bias may account for the conflicting results.

Studies of patients with ankylosing spondylitis suggest an increased risk of colonic cancer among those treated by irradiation. ${ }^{7}$ 
A rise in mortality from colonic cancer (but not rectal cancer) has been observed among the irradiated Japanese populations of Hiroshima and Nagasaki. ${ }^{9}$ Levels of irradiation among these populations, however, probably far exceed therapeutic levels.

Cohort studies have failed to show an increased risk of large bowel malignancy in radiologists and personnel working in atomic installations, but doses of radiation to the bowel in these groups are probably far lower than therapeutic levels. The significance of these studies is therefore difficult to assess."

Case reports provide another source of evidence for a link between therapeutic radiation and large bowel cancer, but strict criteria are necessary before accepting a causal link because of the high incidence of large bowel cancer. Black and Ackerman have proposed the following criteria: (1) a relatively great exposure of radiation to the bowel; (2) a minimum period of 10 years between exposure and tumour development; and (3) radiation induced change in the immediate vicinity of the tumour. "Fewer than a hundred patients in total have been reported and many of these reports fail to fulfil the criteria."

As far as we can determine, this is the first report of two synchronous primary bowel carcinomas developing after pelvic irradiation and the first report of a small bowel carcinoma developing after irradiation. Both tumours fulfil Black and Ackerman's three criteria for a causal link. Large bowel cancer is so common that it is difficult to be completely certain that its development after irradiation is not coincidental. Primary small bowel adenocarcinoma, however, is rare. Although it is not possible with absolute certainty to exclude the small bowel tumour reported here as being a secondary from the large bowel, the evidence presented strongly favours it being a synchronous primary.

\section{References}

1 Palmer JP, Spratt DW. Pelvic carcinoma following irradiation for benign gynecologic diseases. Am J Obstet Gynecol 1956; 72: 497-505.

2 Dickson RJ. Late results of radium treatment of carcinoma of the cervix. Clin Radiol 1972; 23: 528-35.

3 Bailar JC. The incidence of independent tumours among uterine cancer patients. Cancer 1963; 16: 842-53.

4 Corscaden JA, Fertig JW, Gusberg SB. Carcinoma subsequent to the radiotherapeutic menopause. $A m \mathrm{~J}$ Obstet Gynecol 1946; 51: 1-12.

5 Stander RW. Irradiation castration: a follow-up study of results in benign pelvic disease. Obstet Gynecol 1957; 10: 223-9.

6 Smith PG, Doll R. Late effects of $x$-irradiation in patients treated for metropathia haemorrhagia. $\mathrm{Br} J$ Radiol 1976; 49: 224-32.

7 Court Brown WM, Doll R. Mortality from cancer and other causes after radiotherapy for ankylosing spondylitis. Br Med J 1965; 2: 1327-32.

8 Smith PG, Doll R, Radford EP. Cancer mortality among patients with ankylosing spondylitis not given $\mathrm{x}$ ray therapy. Br J Radiol 1977; 50: 728-34.

9 Kato H, Schull WJ. Studies of the mortality of A-bomb survivors. 7. Mortality, 1950-1978: Part I. Cancer mortality. Radiat Res 1982; 90: 395-432.

10 Sandler RS, Sandler DP. Radiation-induced cancer of the colon and rectum: assessing the risk. Gastroenterology $1983 ; 84$ : 51-7.

11 Black WC, Ackerman LV. Carcinoma of the large intestine as a late complication of pelvic radiotherapy. Clin Radiol 1965; 16: 278-81. 